\title{
Advancing research in industrial and organisational psychology - A brief overview of 2013
}

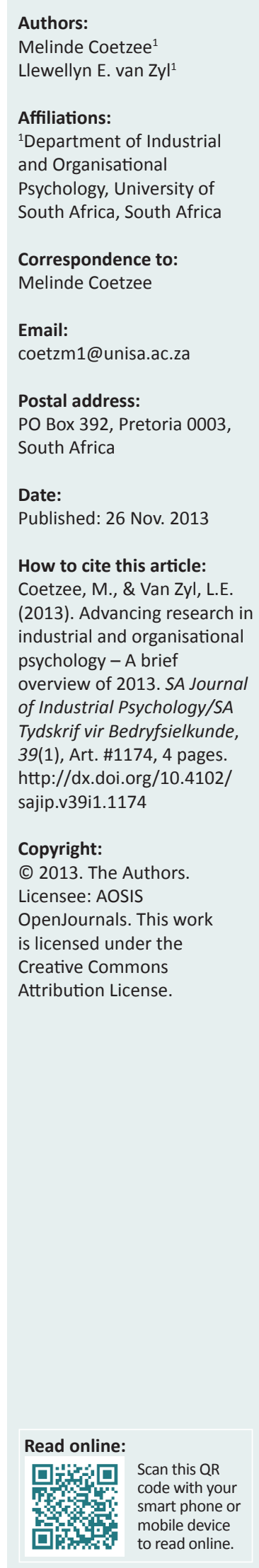

\section{Introduction}

The South African Journal of Industrial Psychology (SAJIP) is a premier southern African journal that focuses on advancing innovative research in the field of industrial and organisational psychology by publishing high-quality manuscripts from both local and international scholars. The editors would firstly like to thank the editorial committee, peer reviewers and the publisher, AOSIS OpenJournals ${ }^{\circledR}$, for their commitment and dedication to ensuring that the manuscripts accepted for publication contribute to the scientific and academic standing and excellence of the SAJIP. Secondly, the editors are grateful for the authors who contributed to the 2013 volume 39(1). The articles published in this volume reflect high-quality original scholarly research endeavours in the field and practice of industrial and organisational psychology.

The articles published showcase research relating to a wide range of themes relevant to the practice of industrial and organisational psychology in the contemporary organisation, for example employee satisfaction and organisational performance in the South African government, employee perceptions of risks and rewards in terms of corporate entrepreneurship participation, biographic and demographic variables as moderators in the prediction of turnover intentions, intention to quit amongst Generation $\mathrm{Y}$ academics, employability attributes and personality preferences of professionals in the business management field, emotional intelligence and leadership styles, engagement and burnout in job demands-resources theory, comprehensibility, manageability and meaningfulness at work, well-being indicators and teacher psychological stress in public schools, work-family conflict and work engagement amongst working mothers, work-related sense of coherence, sense of coherence and job characteristics, emotion-episodes of Afrikaans-speaking employees in the workplace, perceived organisational support in the banking industry, learning programme management in skills development, and construct validity of a development assessment centre.

In the broader African and European contexts, specific themes include agency relations and managed performance in public universities in Uganda, transformational leadership, psychological safety and learning behaviour in work teams in Ghana, psycho-organisational variables and job burnout amongst hotel workers in Nigeria, and measuring work-related sense of coherence in large Swiss companies from diverse economic sectors.

\section{The strategic direction of SAJIP}

The year 2013 witnessed a change in editorship with Professor Gert Roodt (University of Johannesburg, South Africa) stepping out of his role as editor-in-chief. The restructured editorial committee and board of SAJIP are committed to building on the outstanding work and leadership provided by Professor Roodt. A core focus is enhancing the academic and scientific stature and international standing of the journal. During his time as editor-in-chief, Professor Roodt developed and implemented various initiatives to enhance the impact factor and scientific credibility of the journal. These initiatives culminated in an increase in the journal's Global Impact Factor (GIF).

The GIF of a journal is calculated based on:

1. The number of times articles published during a given publication cycle are cited by journals during that period.

2. The total number of 'citable items' published by this journal during the given cycle. ('Citable items' are usually articles, reviews, proceedings or notes, not editorials or letters to the editor.)

The GIF is calculated as point 1 divided by point 2. In 2011, SAJIP's GIF was positioned at 2.45 and increased dramatically to 3.66 in 2012. This implies growth in the national and international impact of SAJIP. One of the key objectives set out by the new editorial committee is to further enhance SAJIP's current GIF and to expand its international stature. Various initiatives in this 
regard are in the process of being implemented. Four of the most significant initiatives are: (1) repositioning the editorial committee and board for broader international exposure, (2) enhancing customer relations with authors, (3) capacity building of 'junior' researchers and reviewers and (4) providing incentives to peer reviewers and section editors.

Firstly, the editorial committee has been expanded to include additional new section editors (locally and internationally) in order to grow SAJIP's capacity to increase its manuscript review turnaround time. Traditionally, SAJIP section editors are known for their scholarly expertise and academic standing in the field of industrial and organisational psychology. They are key to ensuring high-quality peer reviews and that the editorial independence of SAJIP is maintained in the peer review process. Similarly, a number of new section editors were invited to join the SAJIP editorial team based on their high standing in both the national and international research spheres. SAJIP is privileged to welcome the following scholars to the editorial committee:

\section{- Newly appointed section editors}

- Dr Colleen Bernstein(University of the Witwatersrand, South Africa)

- Dr Yannick Griep (Vrije Universiteit Brussel, Belgium)

- Dr Manfred Janik (University of Namibia, Namibia)

- Dr Lené Jorgensen (North-West University, South Africa)

- Dr Eileen Koekemoer (North-West University, South Africa)

- Prof. Anja van den Broeck (Human Relations Research Group, Belgium),

- Prof. Fons van de Vijver (Tilburg University, The Netherlands)

SAJIP is privileged to welcome the following scholars to the editorial board:

- Prof. Marié Wissing (North-West University, South Africa)

- Prof. Fons van de Vijver (Tilburg University, The Netherlands)

- Prof. Gert Roodt (University of Johannesburg, South Africa)

Secondly, the editorial committee, in collaboration with the publisher, AOSIS OpenJournals ${ }^{\circledR}$, has established new processes and practices in order to increase the information flow to authors who submitted manuscripts to SAJIP. The journal's publication platform (website) will be adapted to provide authors with more up-to-date information for tracking the review and publication process of a manuscript.

Thirdly, capacity building initiatives will be implemented for junior researchers and reviewers. This is in order to enhance the research competence level of junior reviewers and to ensure the long-term sustainability of the quality of the journal's reviews. Various initiatives in this regard will be implemented during the next publication cycle.

Fourthly, the journal has introduced an incentive scheme for section editors and peer reviewers. As the majority of our section editors and peer reviewers are professionally registered psychologists and/or health-care practitioners who selflessly donate their personal time to assist in advancing research in the field and enhance the scientific merit of SAJIP, the journal has implemented an honorarium of five Continuing Professional Development and Continuing Education units (CPD/CEUs) for section editors per published manuscript and three CPD/CEUs per manuscript reviewed for peer reviewers registered with the Health Professions Council of South Africa (HPCSA). Although a small token, in relation to the effort and time dedicated to the review process, the editorial committee believes that it is imperative to reward section editors and peer reviewers for the valuable and quality work they perform on the behalf of SAJIP. High-quality reviews of manuscripts are imperative to maintaining and enhancing the scientific merit of the journal and we trust this honorarium provides SAJIP's section editors and peer reviewers with an indication of our sincere appreciation for the valuable time they invest in SAJIP.

Finally, various other initiatives will be implemented during the course of the 2013/2014 publication cycle to enhance the quality, rate and turnaround time of manuscript review and publication and to increase international exposure. Some of these include: (1) submitting the journal for accreditation by the Institute for Scientific Information (ISI), (2) introducing annual special themes, (3) introducing alternative manuscript format types in addition to the full-length original research articles, (4) approaching expert statistical consultants to act on the editorial committee and (5) providing useful feedback to authors regarding their manuscripts and the review and publication process.

\section{Reasons for declining a manuscript for publication in SAJIP}

In relation to the final point above, we would like to take the opportunity to provide the readership with a brief overview of the reason manuscripts are declined for publication in SAJIP. Published manuscripts in a scientific journal are principally regarded as a substantiation of reliable and dependable research. A published article in a given journal is therefore 'endorsed' upon acceptance, which provides credibility to the nature, scope and accuracy of the contents. As a result, manuscript submissions need to be thoroughly scrutinised by the editorial committee and the blind peer review process in order to obtain this proverbial endorsement. Although SAJIP has taken a developmental stance in its editorial policy, some manuscripts are just not publishable. There are a number of reasons why a manuscript can be declined by the journal. Below, some of the major contributing factors are presented.

Reason 1: One of the main reasons for the decline of a manuscript is that it is not positioned in line with the intended scope of the journal. Although some of these articles are well written and positioned, they do not fall completely within the aforementioned scope and would be better positioned with a more relevant journal. The intended scope and focus of the journal is: 'SAJIP is a premier southern African 
journal that focuses on innovative research and scholarship from both local and international sources within the fields of industrial and organisational psychology'. Broadly, industrial and organisational psychology refers to a discipline where psychological theories, models and methodologies are applied in order to understand, predict and describe human behaviour within organisational contexts. The focus of the journal is to develop the discipline of industrial and organisational psychology within the southern African context. Articles falling outside of this scope are declined.

Reason 2: Another reason for a decline relates to the incongruence between the primary research question and the methodology. Further, the research questions and empirical outcomes of the manuscript are misaligned.

Reason 3: Studies in which variables are proverbially 'thrown together' with no clear theoretical relationship between variables or poorly conceptualised arguments are declined from publication. It is imperative to note that arguments should be well presented and in line with the intended scope of the article. A clear argument as to the relationship between the constructs should be presented and supported with appropriate and recent research literature.

Reason 4: Studies that do not clearly articulate the value or contribution of the manuscript in relation to the discipline or profession are declined. A publication in a scientific journal, unlike a master's dissertation, should clearly articulate its originality and indicate the contribution and value added to the respective discipline.

Reason 5: The study lacks psychological depth in its presentation and discussion. As the journal is focused on advancing research in industrial and organisational psychology as an applied field of psychology, it is imperative to always ask the question 'what do the results mean psychologically?' The empirical results should therefore be interpreted and explained in terms of the psychological implications and be integrated with the research literature to substantiate arguments and viewpoints.

Reason 6: Self-plagiarism. Although few in number, there have been instances where authors have published the same study in a different journal or in a peer-reviewed conference proceeding. This is not only unethical, but also fraudulent in nature. Authors are advised to ensure that submissions to the journal are unique and original. The journal employs sophisticated plagiarism detection software which streamlines the detection of (self-)plagiarism. Authors will be informed if plagiarism or self-plagiarism have been detected before publication of a manuscript.

Reason 7: There are errors or flaws in the research methodology or data analysis technique(s) used. When errors or flaws are present in the utilised research methodology or an inappropriate data analysis technique is used to address the proposed research question or research objective, the article may be declined during either the submission or publication process. Some examples of this are: samples are too small to warrant the statistical techniques employed $(n<100)$; the use of parametric or non-parametric statistical techniques are not clearly substantiated in terms of sample and data properties; traditional structural equation modelling is used on small samples $(n<300)$; thematic content analysis is used to 'determine relationships between constructs' (and the like); measuring instruments are not clearly described in terms of reliability and validity. It is important to ensure that the analysis method is appropriate for the intended research design.

Reason 8: Improper rationale. The conceptualisation and presentation of the literature review and problem statement does not warrant the need for the research. In some instances, the literature and problem statement are misaligned or do not highlight the need for the research.

Reason 9: The article lacks novelty and/or originality. A research article submitted to SAJIP should be novel in its contribution to the discipline or in its methodology. Within the South African context, researchers are placed under pressure to publish by various bodies. The result of this is a number of replication studies with no new or original contribution to the field. For example, various established models and theorems are tested within 'various contexts' in order to determine their 'cross-cultural validity, reliability and predictability'. In effect, the sample model or theorem is tested within different samples. The same presentation of the literature and problem statement is presented and the results are usually in line with previous research. Novelty, originality and contribution to the field are therefore lacking. Submissions should provide a clear contribution either in terms of theory building or methodology in order to be considered for publication.

Reason 10: The use of analysis methods that have become obsolete because of new developments in the field. In rare circumstances, an article can be declined based on the obsolete nature of the analysis method. For example, if an article is submitted that solely utilises descriptive statistics or mean comparisons to answer the primary research question. These techniques, although relevant and accurate, have become obsolete in their utilisation as they cannot significantly contribute new knowledge to the discipline nor can the results be generalisable.

Reason 11: Small or inappropriate sample sizes. In both quantitative and qualitative research there are various arguments as to sample procedure and selection. Various supporting and argumentative arguments are also present in relation to the 'appropriate sample size'. Although the journal has no clear 'rule of thumb' pertaining to the sample size of a given study, in some circumstances the sample size may be proverbially too small to significantly answer the research questions, or to generalise the results. Further, the small sample may also not justify the use of a given statistical technique, for example the use of structural equation modelling is inappropriate on samples smaller than 300. Obviously, the sample size is dependent on the actual 
population and as a result there may be exemptions to the norm. Authors should aim to utilise appropriate sampling techniques as well as to obtain an appropriate sample size for analysis and interpretative purposes.

Reason 12: Submitted articles fail to adhere to the publication (manuscript format) guidelines of the journal. Articles need to be presented in the correct style, format and structure. SAJIP has set guidelines for quantitative, qualitative and theoretical articles. Articles need to be presented in line with the proposed guidelines of the given approach. Further, strict adherence to the technical requirements, such as the use of the journal's referencing style, language guidelines, font types (and the like), need to be present.

Reason 13: Incorrect interpretation of results. In various circumstances there is misalignment between the presented results and the interpretation and discussion thereof. It is imperative to ensure that the results are presented and interpreted correctly and that the results section is aligned to the discussion.

Reason 14: No clear improvement in the quality of the manuscript between 're-submissions'. Not all articles are declined on the proverbial 'spot'; some authors are requested to resubmit an article for review. Clear guidelines are presented to the author in an attempt to aid in strengthening the scientific merit of the submission. In some instances, there is no clear improvement in the quality of the first, second, third and even fourth submissions. In these circumstances, the manuscript will be declined.

Although not an exclusive list, these reasons explain some of the factors that contribute to an eventual decline of a manuscript for publication by SAJIP. As the journal and its editorial committee have taken a developmental stance towards manuscripts and publications, we hope that the information outlined in this editorial provide more clarity as to what is ' $\mathrm{NOT}^{\prime}$ expected. In future editorials we plan to highlight what 'IS' expected and will present some examples of 'excellent' articles.

\section{A word of thanks to the 'unsung heroes' of SAJIP}

The number of high-quality submissions we receive yearly, from reputable scholars in the field of industrial and organisational psychology, is a major contributor to the success and standing of the journal. These articles are the result of well-planned and well-executed projects and authors are usually rewarded by their institutions for these publications. These publications not only add scientific credibility to the journal, but also aid in enhancing its stature within the international scientific community.

We do, however, tend to forget that these manuscripts go through a number of processes before they are published. For the most part, scholars involved with the process are rewarded in some way. For authors, there are substantial subsidies from the Department of Higher Education (South Africa). For the editorial committee, there are some benefits for their association with and role in the journal. For the publisher (AOSIS OpenJournals ${ }^{\circledR}$ ), there is an income stream and salaries for those involved. However, we tend to forget a crucial part of this process: peer reviewers. SAJIP's peer reviewers are the 'unsung' heroes of the journal, who voluntarily (without payment, recognition or direct benefit) invest their time and expertise to contribute to the success of the journal.

We do need to acknowledge that SAJIP relies heavily upon the voluntary knowledge, skills and expertise of scholars who review manuscripts. These reviewers contribute equally to the success of the journal and also the scientific merit and quality of the finally published articles. More often than not, these individuals are under high pressure from the journal to complete high-quality reviews within a given time frame (despite their own normal work-related demands). The editorial committee and the publisher would like to extend their sincere gratitude to these 'unsung heroes' who selflessly contribute to the success of the journal. We are humbled by your continued investment in the success and stature of SAJIP.

\section{Concluding remarks}

On a final note, we trust that the contributions and insights provided by the articles in this volume will create momentum for future research in the field of ind ustrial and organisational psychology and serve as valuable scholarly resources for researcher and practitioners in the field. We also hope that the information about the strategic direction of SAJIP and factors contributing to the decline of submissions will contribute to the quality and number of new submissions. We would also like to invite you to continue to consider SAJIP as the journal of choice for your publications in order to further advance research in and encourage discourse on the field, profession and practice of industrial and organisational psychology. 